\title{
Hyaluronan-modified superparamagnetic iron oxide nanoparticles for bimodal breast cancer imaging and photothermal therapy
}

This article was published in the following Dove Press journal:

International Journal of Nanomedicine

23 December 2016

Number of times this article has been viewed

\author{
Rui-Meng Yangl,* \\ Chao-Ping $\mathrm{Fu}^{2}, *$ \\ Jin-Zhi Fang' \\ Xiang-Dong $\mathrm{Xu}{ }^{\prime}$ \\ Xin-Hua Wei' \\ Wen-Jie Tang' \\ Xin-Qing Jiang' \\ Li-Ming Zhang ${ }^{2}$
}

'Department of Radiology, Guangzhou First People's Hospital, Guangzhou

Medical University, ${ }^{2}$ School of

Materials Science and Engineering,

School of Chemistry, Sun Yat-sen

University, Guangzhou, China

*These authors contributed equally to this work

\section{Correspondence: Xin-Qing Jiang Department of Radiology, Guangzhou First People's Hospital, Guangzhou Medical University, \#I Panfu Road, Guangzhou 510180, China Tel +862081044902 Email jiangxinqing888@163.com \\ Li-Ming Zhang \\ School of Materials Science and Engineering, School of Chemistry, Sun Yat-sen University, \#I35 Xingang West Road, Guangzhou 510275, China Tel +8620 84II 2454 \\ Email ceszhlm@mail.sysu.edu.cn}

\begin{abstract}
Theranostic nanoparticles with both imaging and therapeutic abilities are highly promising in successful diagnosis and treatment of the most devastating cancers. In this study, the dual-modal imaging and photothermal effect of hyaluronan (HA)-modified superparamagnetic iron oxide nanoparticles (HA-SPIONs), which was developed in a previous study, were investigated for CD44 HA receptor-overexpressing breast cancer in both in vitro and in vivo experiments. Heat is found to be rapidly generated by near-infrared laser range irradiation of HASPIONs. When incubated with CD44 HA receptor-overexpressing MDA-MB-231 cells in vitro, HA-SPIONs exhibited significant specific cellular uptake and specific accumulation confirmed by Prussian blue staining. The in vitro and in vivo results of magnetic resonance imaging and photothermal ablation demonstrated that HA-SPIONs exhibited significant negative contrast enhancement on $\mathrm{T}_{2}$-weighted magnetic resonance imaging and photothermal effect targeted CD44 HA receptor-overexpressing breast cancer. All these results indicated that HA-SPIONs have great potential for effective diagnosis and treatment of cancer.
\end{abstract}

Keywords: iron oxide nanoparticles, surface functionalization, bioactive glycosaminoglycan, magnetic resonance imaging, cellular uptake, breast carcinoma

\section{Introduction}

In recent years, near-infrared (NIR) laser-induced photothermal therapy (PTT) based on the nanotechnology, which can deliver a specific amount of photoenergy directly into the tumor site to increase treatment efficiency and meanwhile minimize the damage to the surrounding healthy tissues, has emerged as an appealing and minimally invasive therapeutic strategy for cancer treatment. Noble metal-based nanostructures, such as gold nanospheres, ${ }^{1,2}$ gold nanorods, ${ }^{3,4}$ gold nanoshells, ${ }^{5}$ gold nanocages, ${ }^{6} \mathrm{Cu}$-based semiconductor nanoparticles, ${ }^{7}$ and carbon nanotubes ${ }^{8}$ are the chief nanomaterials that have been well prepared and demonstrated powerful NIR laser-induced PTT efficacy. However, their promising clinical applications were largely limited due to the innate toxicity and unknown long-term toxicity of these aforementioned PTT agents.

Superparamagnetic iron oxide nanoparticles (SPIONs), which possess unique properties including non-toxicity, magnetic properties, chemical stability, and biocompatibility, ${ }^{9-11}$ have also been recently discovered to have photothermal effect and therefore could be used as an alternative PTT material. ${ }^{9,12-15}$ Chen et al ${ }^{14}$ reported that the highly crystallized iron oxide nanoparticles administered through the tail vein for PTT as effective and biodegradable mediators. These injected nanoparticles are likely to be applied for PTT as a result of the enhanced permeability and retention (EPR) effect and is cleared from the body through urinary excretion without long-term toxicity. cc. hereby accept the Terms. Non-commercial uses of the work are permitted without any further permission from Dove Medical Press Limited, provided the work is properly attributed. For permission for commercial use of this work, please see paragraphs 4.2 and 5 of our Terms (https://www.dovepress.com/terms.php). 
As already known, SPIONs were also used in clinical studies as an excellent $\mathrm{T}_{2}$-weighted magnetic resonance imaging (MRI) contrast agent. Hence, SPIONs basis theranostic agent, which could combine diagnostic MRI and NIR laser-induced PTT therapeutic capabilities into one single entity, would be a great value for optimal medical outcome. ${ }^{16-18}$ However, in many cases, nanoparticles are simultaneously transferred to the reticuloendothelial system (RES) of liver and spleen, thus significantly reducing their treatment efficiency on tumor cells. Therefore, there has been a growing demand to develop various tumor-targeted SPIONs systems for the PTT and MRI of tumor sites.

A previous study ${ }^{19}$ developed a modified SPIONs naturally occurring glycosaminoglycan (hyaluronic acid, HA), with specific biological recognition to human cancers overexpressing CD44 HA receptors, and this study set out to evaluate the MRI ability and photothermal effect of HA-SPIONs which the previous study had developed for targeted breast cancer to investigate its possibility to be used as a theranostic agent. For this purpose, the photothermal conversion effect was initially studied on dry HA-modified SPIONs powders. Then, HA-modified SPIONs were applied on CD44 HA receptors overexpressing MDA-MB-231 breast tumor cells to investigate the specific cellular uptake. Additionally, MRI and photothermal efficacy of HA-modified SPIONs were investigated with in vitro and in vivo experiments.

\section{Experimental section Materials}

Ferric chloride hexahydrate $\left(\mathrm{FeCl}_{3} \cdot 6 \mathrm{H}_{2} \mathrm{O}\right)$, ferrous sulfate $\left(\mathrm{FeSO}_{4}\right)$, ammonium hydroxide, 1-ethyl-3-[3(dimethylamino)-propyl]carbodiimide, $N$-hydroxysuccinimide, and fluorescein isothiocyanate were purchased from Aladdin Reagent Database Inc. (Shanghai, China). HA (MW $320 \mathrm{kDa}$ ) was purchased from Freda Biochem Co., Ltd. (Shandong, China). 3-Aminopropyltrimethoxysilane (APTMS, pH 7.4) was purchased from Sigma-Aldrich (St Louis, MO). Fetal bovine serum (FBS, 10\%), Dulbecco's Modified Eagle's Medium (DMEM) were purchased from Hyclone/Thermo Scientific (Waltham, MA, USA). All other chemicals and reagents were of analytical grade.

\section{Cell culture}

MDA-MB-231 cells, purchased from Land Biology Technology Company Co., Ltd. (Guangzhou, Guangdong, China), were cultured in DMEM supplemented with $10 \%$ of FBS, antibiotics $(100 \mu \mathrm{g} / \mathrm{mL}$ streptomycin and $100 \mathrm{U} / \mathrm{mL}$ penicillin) at $37^{\circ} \mathrm{C}$ in a humidified atmosphere with $5 \%$ of
$\mathrm{CO}_{2}$. Cells were both harvested by the use of trypsin and resuspended in a fresh complete medium before plating.

\section{Animals}

All animal experiments were performed under ethical approval from and in agreement with the guidelines of the Institutional Animal Care and Use Ethics Committee of Sun Yat-sen University and also in accordance with the policy of the National Ministry of Health. Tumors were induced by subcutaneous injection of MDA-MB- 231 cells $\left(1 \times 10^{7}\right.$ cells per mouse $)$ into the right flank of BALB/c female nude mice (4-5 weeks, 18-20 g).

\section{Synthesis of HA-SPIONs}

The hyaluronan-targeted SPIONs were synthesized according to the method of Yang et $\mathrm{al}^{19}$ by the chemical conjugation of hyaluronan, as reported in a recent publication.

\section{Measurements of photothermal conversion effect}

The dry powders obtained $(10 \mathrm{mg})$ were placed on the parafilm (Polysciences Inc, Warrington, USA; thickness: $0.127 \mathrm{~mm}$ ). Lasers (Xi'an Minghui Optoelectronics Tech. Co., Ltd., Xi' an, China) with wavelength of $808 \mathrm{~nm}$ (power density, $1 \mathrm{~W} / \mathrm{cm}^{2}$; spot area, $10 \times 8 \mathrm{~mm}$ ) were used to irradiate the nanoparticle powders through the parafilm at room temperature. As a control, the parafilm was irradiated with the same lasers as described earlier.

To accurately monitor the temperature changes in samples upon laser irradiation, the SPIONs and HA-SPIONs powders were dissolved in water $(10 \mathrm{~mL})$ in a round-bottomed flask to get the nanoparticle aqueous suspension of different concentrations $(10 \mathrm{~mL}, 100-500 \mu \mathrm{g} / \mathrm{mL})$. The $808 \mathrm{~nm}$ lasers were then used to irradiate the solutions for 1, 2, 3, 5, 7, or $9 \mathrm{~min}$, respectively. As controls, the temperatures of distilled water irradiated by the same laser as described earlier were measured. All the experiments were conducted in triplicate.

\section{In vitro cellular uptake studies - Perl's Prussian blue staining}

MDA-MB- 231 cells at a density of $5 \times 10^{5}$ cells/well were seeded onto the 12-well plate and incubated for $24 \mathrm{~h}$, and $1 \mathrm{~mL}$ of the DMEM containing SPIONs or HA-SPIONs (Fe concentration was fixed to be $40 \mu \mathrm{g} / \mathrm{mL}$ ) were added and incubated at $37^{\circ} \mathrm{C}$ for $24 \mathrm{~h}$. Then Perl's Prussian blue staining assay was performed according to the method which was published earlier. ${ }^{19}$ The wells without SPIONs or HA-SPIONs were used as control. Perl's Prussian blue staining images were taken on an Olympus BX51 optical microscope (Olympus Corp, Tokyo, Japan). 


\section{In vitro MRI of MDA-MB-23I cells internalized with nanoparticles}

In vitro MRI was performed with MDA-MB-231 cells after incubation with SPION or HA-SPION nanoparticle at the same Fe concentrations $(40 \mu \mathrm{g} / \mathrm{mL})$ for $24 \mathrm{~h}$. After rinsing with PBS, the cells were trypsinized and counted. $1 \times 10^{4}$, $1 \times 10^{5}$, or $1 \times 10^{6}$ cells/well were placed into $2 \mathrm{~mL}$ Eppendorf tubes. After centrifugation and resuspension in $1 \mathrm{~mL}$ PBS (containing $0.5 \%$ agarose), the Eppendorf tubes were placed into a 3.0 T MRI scanner (Verio, Siemens, Erlangen, Germany) at room temperature for MRI. $\mathrm{T}_{2}$ mapping sequence (TR: 1,000 ms, TE: 13.8/27.6/41.4/55.2/69.0 ms, flip angle: $180^{\circ}$, slice thickness: $3.0 \mathrm{~mm}$, matrix $444 \times 448$ ) was used to measure $\mathrm{T}_{2}$ transverse relaxation time.

\section{In vitro photothermal ablation of tumor cells}

The MDA-MB-231 cells with a density of $1 \times 10^{5}$ per well were seeded into a 12 -well plate and incubated with DMEM containing HA-SPIONs $(200 \mu \mathrm{g} \mathrm{Fe} / \mathrm{mL})$ at $37^{\circ} \mathrm{C}$. After $24 \mathrm{~h}$ of incubation, cells were washed three times with PBS and irradiated with the $808-n m$ NIR laser at an output power density of $2 \mathrm{~W} / \mathrm{cm}^{2}$ for $10 \mathrm{~min}$. Then, the cells were stained with calcein AM (calcein acetoxymethyl ester) and propidium iodide (PI) to verify the photothermal effect on cancer cells. In addition, MDA-MB-231 cells were stained with 4',6-diamidino-2-phenylindole (DAPI) for $15 \mathrm{~min}$ and then imaged through Olympus IX-71 optical microscope (Olympus, Tokyo, Japan).

\section{In vitro photothermal toxicity of HA-SPIONs}

MDA-MB-231 cells $\left(1 \times 10^{4}\right.$ cells per well) were seeded in 96-well plates and incubated for $24 \mathrm{~h}$ to allow for cell adhesion, and then the medium was replaced with serum-free medium containing various concentrations of HA-SPIONs. After further $24 \mathrm{~h}$ of incubation, the cells were washed three times with PBS, and the medium was replaced with DMEM. The cells were then irradiated by an 808-nm NIR laser with an intensity of $2 \mathrm{~W} / \mathrm{cm}^{2}$ for $10 \mathrm{~min}$, while cells without irradiation were used as control. The cell viabilities were determined $1 \mathrm{~h}$ after photothermal treatment using the methyl thiazolyl tetrazolium (MTT) assay. Briefly speaking, $20 \mu \mathrm{L}$ of MTT (Promega, Madison, WI, USA) in a concentration of $5 \mathrm{mg} / \mathrm{mL}$ was added to each well and then the cells were incubated for another $4 \mathrm{~h}$ at $37^{\circ} \mathrm{C}$. Finally, formazan crystals were solubilized by $150 \mu \mathrm{L}$ of dimethyl sulphoxide, and the absorbance was measured with an ELX 800 microplate reader (BioTek, Winooski, VT, USA) at a wavelength of $490 \mathrm{~nm}$. Cell viability was calculated and compared with the untreated control. It was determined from the ratio of absorbance values for irradiated cells to those for "MDA-MB-231 cells + HASPIONs" non-irradiated cells.

\section{In vivo tumor $M R I$}

In vivo MRI was performed using a 3.0 T MRI scanner (Verio; Siemens, Erlangen, Germany) equipped with a soft coil at room temperature. Tumor-bearing mice were imaged using a spin echo sequence $(\mathrm{TR}=4,500 \mathrm{~ms}, \mathrm{TE}=82 \mathrm{~ms}$, matrix size $=256 \times 256$, thickness $=2 \mathrm{~mm}$ ). Images were taken before and after tail vein injection of HA-SPIONs at different time points. $\mathrm{T}_{2}$-weighted images were analyzed using a program developed in MATLAB ${ }^{\circledR}$.

\section{In vivo PTT}

A total of 24 mice with similar sized tumor (length $\approx$ width $\approx 1 \mathrm{~cm}$ ) were selected for PTT. The tumor-bearing mice were divided into six groups, namely blank control, laser only, SPIONs only, HA-SPIONs only, SPIONs + laser, and HA-SPIONs + laser groups. For magnetic NP group, mice were injected with SPIONs or HA-SPIONs $(20 \mathrm{mg} / \mathrm{kg}$ body weight) through tail vein. Mice without any treatment were blank control group. In the three irradiation groups, tumors were irradiated with the $808 \mathrm{~nm}$ NIR laser at a power density of $2 \mathrm{~W} / \mathrm{cm}^{2}$ for $10 \mathrm{~min}$ every $24 \mathrm{~h}$ for 8 days after injection. The remaining three groups were not laser irradiated. The tumor morphologies were recorded with a digital color camera during the treatment. The tumor sizes were measured at the widest width and along the perpendicular length using a caliper, and they were calculated using the formula tumor volume $=($ tumor length $) \times(\text { tumor width })^{2} / 2$.

\section{Histological analysis of tumor tissues}

All the mice were killed, and the tumors were resected 12 days after the injection of nanoparticles. Tumor tissue blocks were then processed through graded alcohols and embedded in paraffin. All paraffin-fixed blocks were sectioned from the center at a thickness of $4 \mu \mathrm{m}$. Sections of tumor tissue were placed onto glass slides and terminal deoxynucleotidyl transferase biotindUTP nick-end labeling (TUNEL assay) was performed with ApopTag Apoptosis Kit (Millipore, Billerica, MA, USA) to analyze apoptotic cells according to the manufacturer's protocol. Images of the tissue sections were obtained through Olympus IX-71 optical microscope (Olympus, Tokyo, Japan). 


\section{Results and discussion \\ Measurement of photothermal conversion effect}

The photothermal conversion effect was initially studied on dry SPIONs and HA-SPIONs powders, which were placed on the parafilm. Lasers of wavelength $808 \mathrm{~nm}$ (power density, $1 \mathrm{~W} / \mathrm{cm}^{2}$; spot area, $10 \times 8 \mathrm{~mm}$ ) were used to irradiate the nanoparticle powders through the parafilm at room temperature as schematically shown in Figure 1. As control, the parafilm was irradiated with the same lasers as described earlier. Upon laser irradiation, the dry powder generates sufficient heat and rapidly burns out a considerable area of the parafilm that is directly in contact with the powder within $\sim 30 \mathrm{~s}$ (Figure 1). Obvious differences in photothermal effects were not found in SPIONs and HA-SPIONs. These results indicated sufficient photothermal effects of SPIONs and HA-SPIONs activated by NIR laser irradiation.

The NIR absorbance of SPIONs allowed for effective photothermal heating of solutions with low concentrations of HA-SPIONs. At low HA-SPION concentration, $100 \mu \mathrm{g} / \mathrm{mL}$,
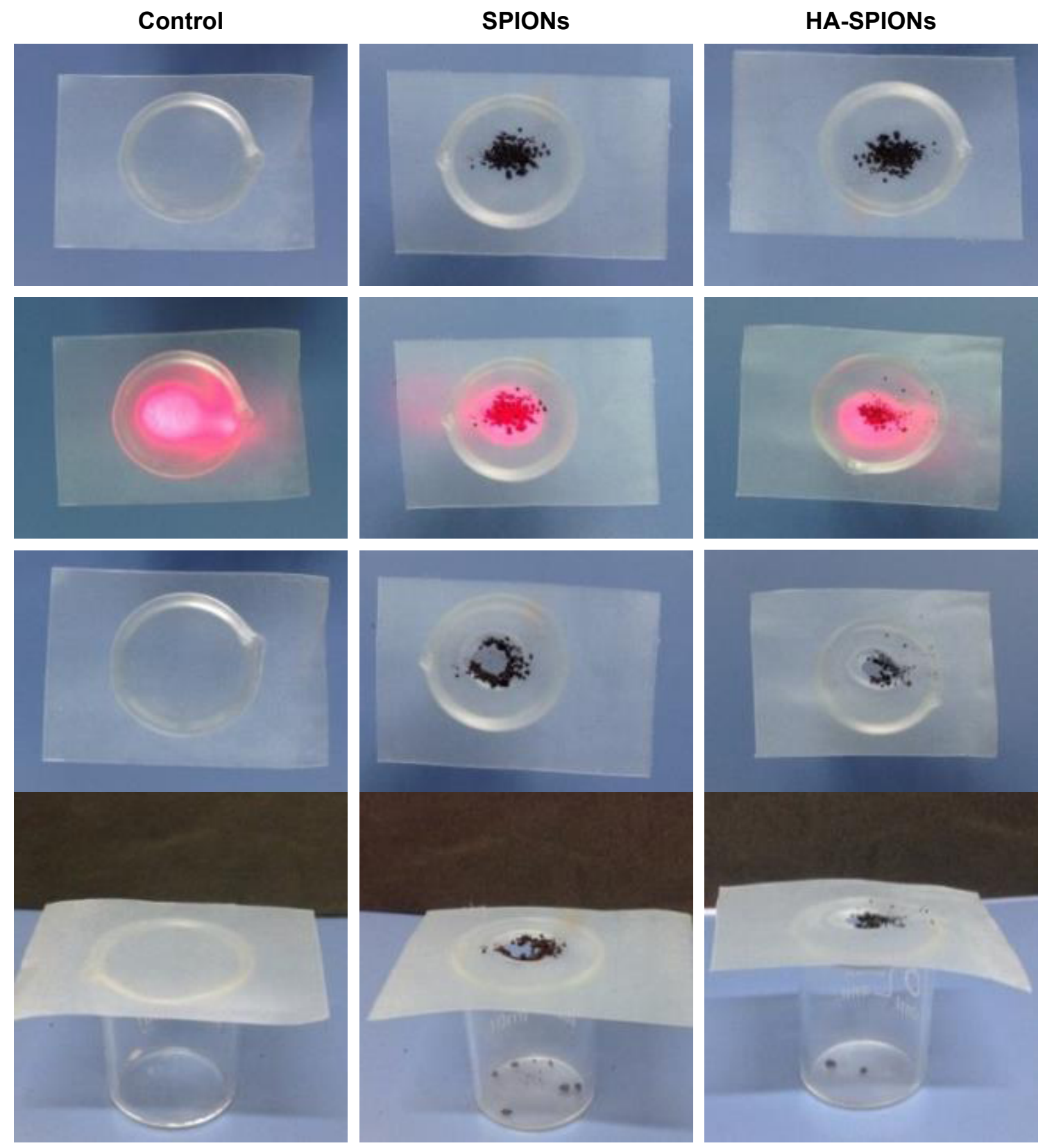

Figure I Photothermal effect of HA-SPION dry powder and its solution induced by $808 \mathrm{~nm}$ laser irradiation. Abbreviation: HA-SPION, hyaluronan-modified superparamagnetic iron oxide nanoparticle. 

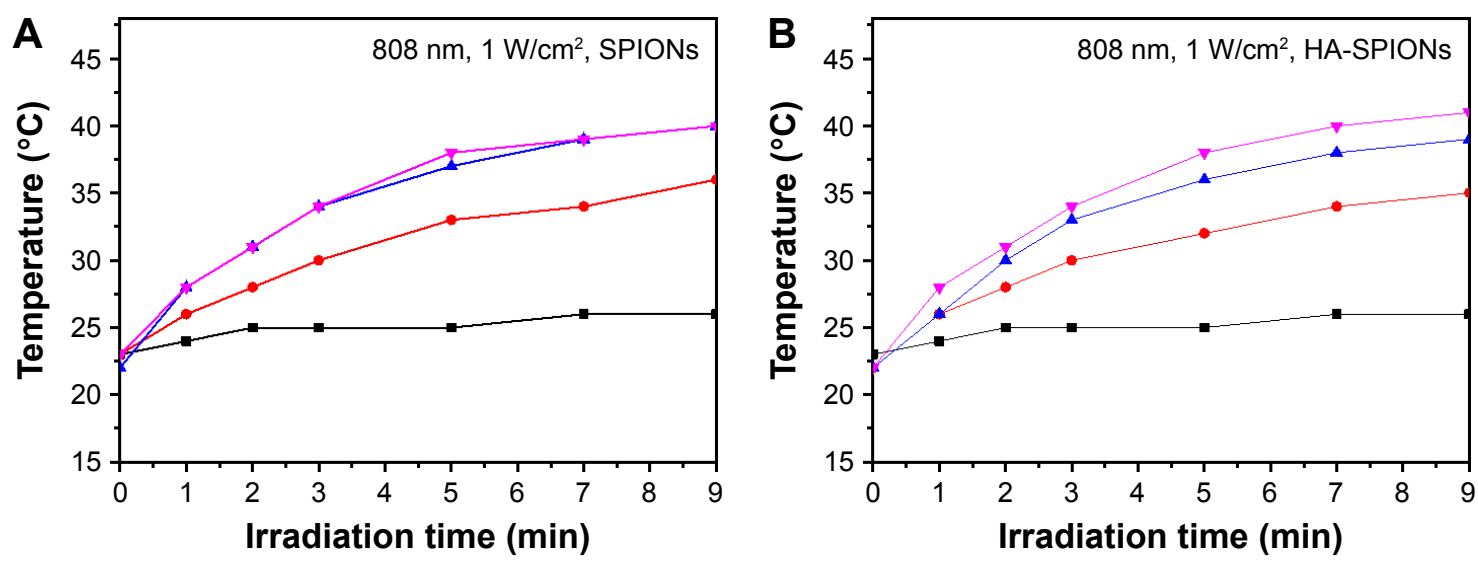

$\rightarrow-$ Water $\rightarrow 100 \mu \mathrm{g} / \mathrm{mL} \longrightarrow 200 \mu \mathrm{g} / \mathrm{mL} \longrightarrow 500 \mu \mathrm{g} / \mathrm{mL}$

Figure 2 Photothermal effect of SPIONs (A) and HA-SPIONs (B) at different concentrations (I00, 200, and $500 \mu \mathrm{g} / \mathrm{mL})$ in aqueous solution under NIR laser irradiation $\left(808 \mathrm{~nm}, \mathrm{I} \mathrm{W} / \mathrm{cm}^{2}\right)$ compared with the distilled water.

Abbreviations: HA-SPION, hyaluronan-modified superparamagnetic iron oxide nanoparticle; NIR, near-infrared.

rapid photothermal heating occurred upon irradiation by a low power $808 \mathrm{~nm}$ laser at $1 \mathrm{~W} / \mathrm{cm}^{2}$ (Figure 2B). Temperatures above the photoablation limit of $40^{\circ} \mathrm{C}$ were readily reached within $10 \mathrm{~min}$ of irradiation. In contrast, under the same concentration and laser irradiation, a solution of SPIONs can reach the same temperature (Figure 2A). Both the SPIONs and HA-SPIONs solutions exhibited a concentration-dependent photothermal heating effect.

\section{In vitro cellular uptake studies - Perl's Prussian blue staining}

The relevance of the prepared HA-SPIONs as a successful candidate for multifunctional nanoprobes is also dependent on the delivery of the magnetic materials inside cellular environment. ${ }^{20}$ Hence, internalization of the HA-SPIONs was confirmed by Prussian blue staining, a method that gives a characteristic blue color in the presence of ferric ions. The results of the staining studies indicate that the intracellular presence of $\mathrm{Fe}_{3} \mathrm{O}_{4}$ can be visualized by the positive blue spots in MDA-MB-231 cells (Figure 3C). However, similar staining studies on MDA-MB-231 cells with the treatment of SPIONs did not result in any blue spots (Figure 3B). Furthermore, control studies with untreated cells did not result in any positive blue spots (Figure 3A). These results showed receptor-mediated endocytosis, especially an interaction between HA and CD44 HA receptors, identified as the principal cellular uptake mechanism of the HA-based self-assembled nanoparticle.

\section{In vitro MRI of MDA-MB-23 I cells internalized with nanoparticles}

The MDA-MB-231 cell labeling by SPIONs or HA-SPIONs was confirmed by MRI. From the MR image of MDA-MB-231 cells with internalized HA-SPIONs (Figure 4), $\mathrm{T}_{2}$ transverse relax times were significantly decreased in HA-SPIONtreated cell pellets, compared with that of those treated with SPIONs. It was consistent with the results of in vitro cellular uptake Perl's Prussian blue staining studies. The results indicated that SPIONs were difficult for MDA-MB-231 cells to internalize, and further suggested that HA-SPIONs
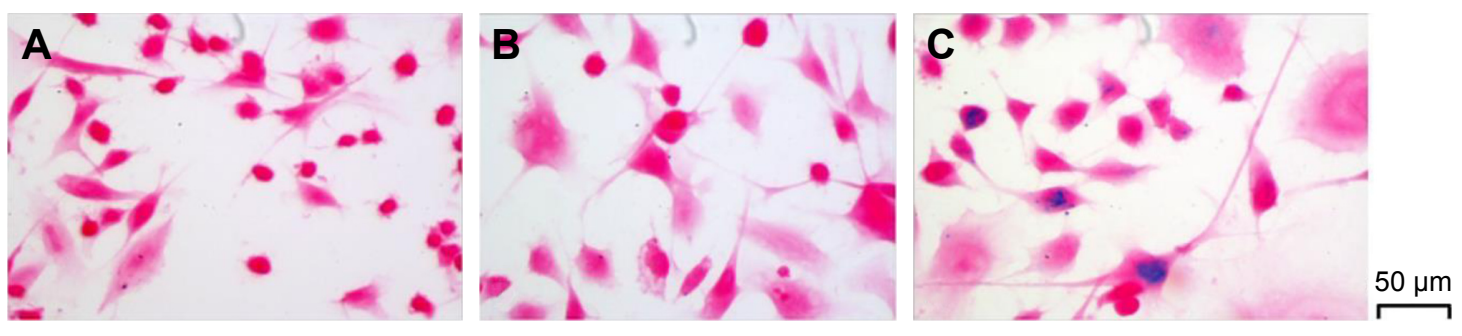

Figure 3 Prussian blue staining images $(\times 400)$ of MDA-MB-23I cells; MDA-MB-23I cells only (A), MDA-MB-23I cells after $24 \mathrm{~h}$ of incubation with SPIONs $($ B), and MDA-MB-23I cells after $24 \mathrm{~h}$ of incubation with HA-SPIONs (C).

Abbreviation: HA-SPION, hyaluronan-modified superparamagnetic iron oxide nanoparticle. 


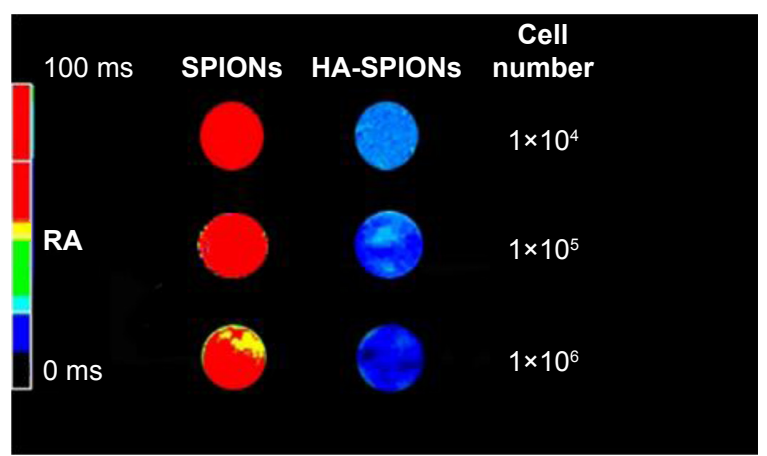

Figure $4 T_{2}$ mapping MR image of pellets of MDA-MB-23I cells after incubation with SPIONs or HA-SPIONs for $24 \mathrm{~h}$. The cell number in each Eppendorf tube was $1 \times 10^{4}, 1 \times 10^{5}$, or $1 \times 10^{6}$, respectively.

Abbreviations: HA-SPION, hyaluronan-modified superparamagnetic iron oxide nanoparticle; MR, magnetic resonance.

could be used as a diagnostic $\mathrm{T}_{2}$-negative $\mathrm{MRI}$ contrast agent targeted for CD44 HA-receptor overexpressing MDA-MB231 breast tumor cells.

\section{In vitro photothermal ablation of tumor cells}

To verify the visible photothermal ablation effect of HASPIONs on cell viability, viable cells staining with calcein AM and dead cells staining with PI were performed using fluorescence microscopy in this study. After laser exposure of $2 \mathrm{~W} / \mathrm{cm}^{2}$ for $10 \mathrm{~min}$, only the HA-SPION-combined laser group (Figure 5A, image d) showed faint red fluorescent region indicating the dead cells that underwent photothermal destruction. In comparison, other samples (Figure 5A, images $\mathrm{a}-\mathrm{c})$ showed green color in the entire area, suggesting that HA-SPIONs only or a NIR laser only would not induce cell death. This result indicated that HA-SPIONs would cause cell death through photothermal effects combined with NIR laser irradiation. In addition, DAPI staining has confirmed the observation of this study. Obvious karyorrhexis, karyopyknosis, and karyolysis could only be seen in the group of HA-SPION-combined laser treatment (Figure 5B, image d) compared with the other groups (Figure 5B, images a-c). These results also demonstrated that tumor cell deaths may not have been influenced solely by magnetic nanoparticle application or laser irradiation.

\section{In vitro photothermal toxicity of HA-SPIONs}

In order to further investigate the in vitro photothermal cytotoxicity of HA-SPIONs, cell viability for MDA-MB-231 cells incubated with HA-SPIONs of different concentrations with or without laser irradiation was determined by the standard MTT assay. After incubation with increasing concentrations of HA-SPIONs, cell viability was accordingly inhibited after exposure of NIR laser irradiation (Figure 6). However, cell viability of MDA-MB-231 cells was not inhibited as expected without laser irradiation. This result also demonstrated that the HA-SPIONs could localize and kill the tumor cells effectively through photothermal effects in vitro.

\section{In vivo $\mathrm{MRI}$}

In vivo tumor-targeted MRI capability of HA-SPIONs as a $\mathrm{T}_{2}$-weighted $\mathrm{MR}$ contrast agent was investigated using MDA-MB-231 tumor-bearing mice (Figure 7). HA-SPIONs generated significantly negative contrast enhancement in tumor tissues, resulting in darkening of the tumor site on $\mathrm{T}_{2}$-weighted MR images by shortening the transverse relaxation time $\mathrm{T}_{2}$ of surrounding water protons. $\mathrm{T}_{2}$-weighted $\mathrm{MR}$
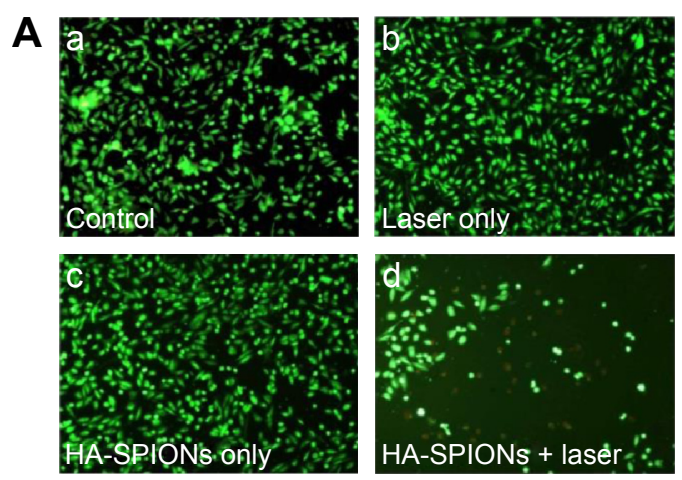
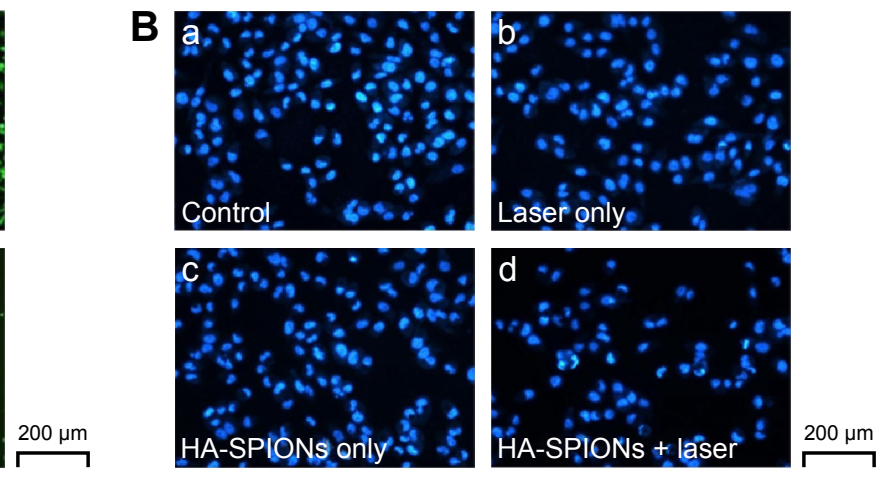

Figure 5 Fluorescence images of calcein AM + PI stained (A) and DAPI stained (B) MDA-MB-23I cells upon different experimental conditions: (a) control; (b) NIR laser $\left(808 \mathrm{~nm}, 2 \mathrm{~W} / \mathrm{cm}^{2}\right)$ treatment only; (c) HA-SPIONs only; and (d) HA-SPIONs combined with NIR laser treatments.

Abbreviations: HA-SPION, hyaluronan-modified superparamagnetic iron oxide nanoparticle; PI, propidium iodide; DAPI, 4',6-diamidino-2-phenylindole; NIR, nearinfrared. 


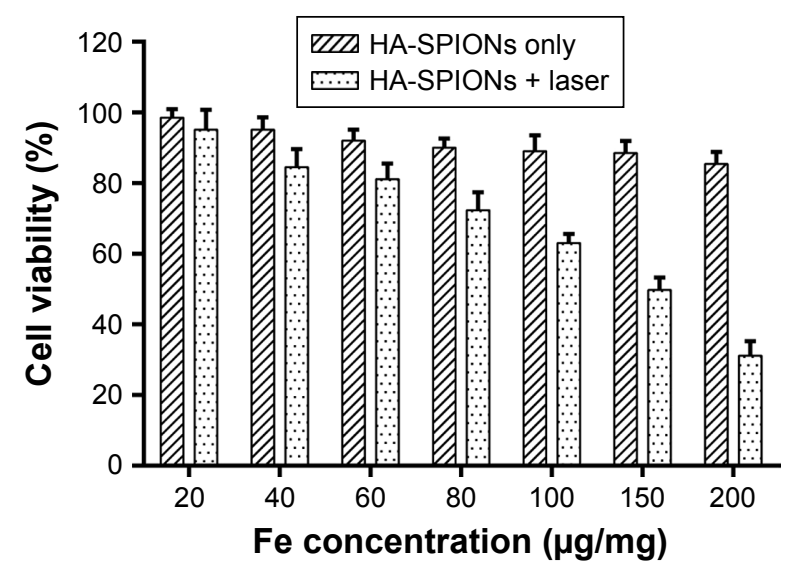

Figure 6 Cell viability after treatment with different concentrations of HA-SPIONs with or without NIR laser exposure.

Abbreviations: HA-SPION, hyaluronan-modified superparamagnetic iron oxide nanoparticle; NIR, near-infrared.

signals at the tumor site gradually decreased, reaching the maximal negative contrast enhancement of $\sim 40 \%$ at $90 \mathrm{~min}$ post-injection. After $24 \mathrm{~h}$ post-injection, the whole tumor area was observed mildly darker than that before administration, suggesting that part of HA-SPIONs still remains at the tumor site. These results demonstrated that HA-SPIONs could be used for targeted MRI of MDA-MB-231 tumors in vivo.

\section{In vivo PTT}

Further in vivo PTT was investigated using HA-SPIONs on female nude mouse xenograft models. As shown in Figure 8, the growth of mice tumors were significantly suppressed after injected with SPIONs or HA-SPIONs, followed by irradiation with the laser. In contrast, mice tumors in blank control, laser only, SPIONs only, and HA-SPIONs only groups grew markedly over time.

In addition, as exhibited in Figure 9, a charring spot appears on the tumor site in SPIONs + laser and HA-SPIONs + laser groups, indicating hemorrhage caused by photothermal heat generated by the $\mathrm{Fe}_{3} \mathrm{O}_{4}$ nanoparticles. Moreover, tumors in the HA-SPIONs + laser group exhibited more significant effect of PTT than SPIONs group, leaving black scars at the original tumor sites without reoccurrence. The results indicated that HA-SPIONs + laser group displayed more effective photothermal ablation via active targeting of CD44 HA receptor than SPIONs + laser group via passive tumor targeting the so-called EPR effect. However, no dark spots were observed in any tumor site in blank control, laser only, SPIONs only and HA-SPIONs only groups. These results clearly showed evidence of tumor growth inhibition by magnetic NP PTT, and the tumor growth could not be affected by either magnetic NPs or laser irradiation. Thus, PTT is a promising approach for MRI-guided tumor ablation.

\section{Histological analysis of tumor tissues}

Tumor-bearing mice injected with PBS or magnetic NPs with and without laser NIR irradiation were examined. The apoptosis of tumor cells induced by photothermal effect of magnetic NPs was confirmed by TUNEL assays. Generally, the apoptotic cells were stained brown, whereas the robust and viable cells stained blue. Many cells of the mouse tumor

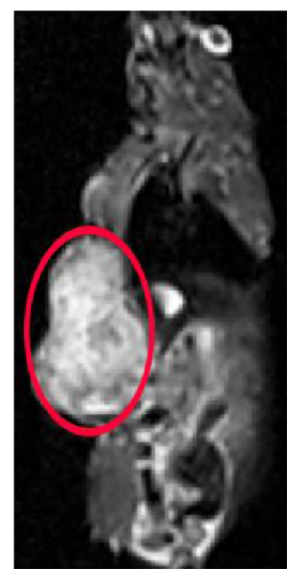

Pre-injection

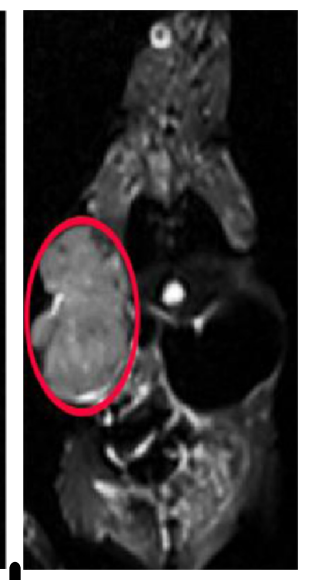

$30 \mathrm{~min}$

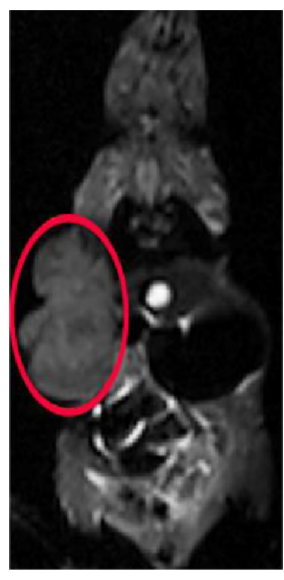

$90 \mathrm{~min}$

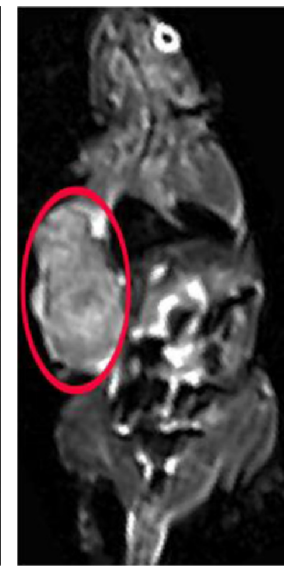

$24 \mathrm{~h}$

Post-injection

Figure $7 \mathrm{~T}_{2}$-weighted MR images of MDA-MB-23I tumor (areas in red circle) bearing mice before and after intravenous injection with HA-SPIONs. Abbreviations: HA-SPION, hyaluronan-modified superparamagnetic iron oxide nanoparticle; MR, magnetic resonance. 


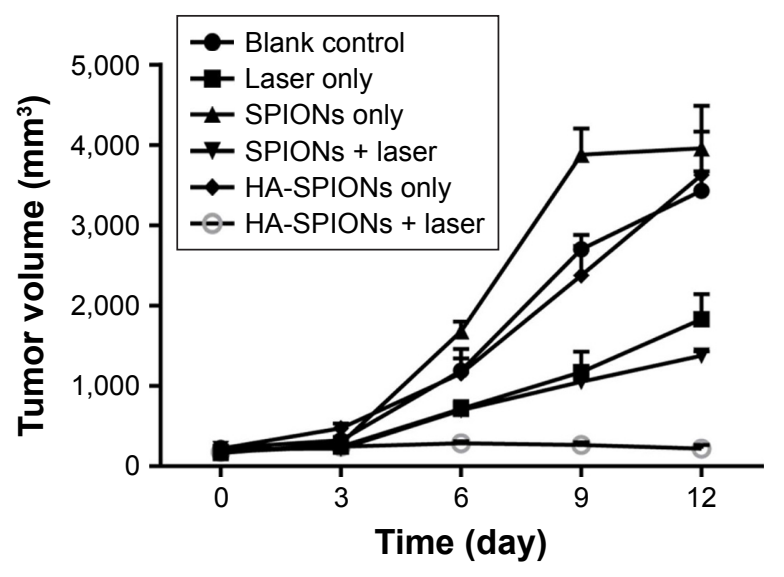

Figure 8 Tumor growth curves with and without laser irradiation treatment. Abbreviation: HA-SPION, hyaluronan-modified superparamagnetic iron oxide nanoparticle.

injected with the magnetic NPs exhibited brown staining after laser irradiation, which indicated dead or apoptotic cells (Figure 10). Therefore, the growth of tumors synchronously treated with magnetic NPs combined with laser irradiation was clearly inhibited. Interestingly, magnetic NPs combined with laser irradiation treatment showed higher damaged cells in HA-SPION group due to active targeting of CD44 HA receptor than SPIONs group, which is consistent with in vivo PTT results. However, the cells of mouse without laser irradiation showed blue staining, which indicated that these cells were not affected.

Histology analysis of tumor tissues further demonstrated that the tumor cells are significantly destroyed by the HASPION laser irradiation treatment.

\section{Conclusion}

In the current study, HA-SPIONs exhibited exquisite ability to targeted MRI and highly effective PTT induced by near-infrared laser irradiation for CD44 HA receptors highly expressing breast cancer in both in vitro and in vivo studies. The photothermal effect of magnetic iron oxide may be well utilized as an efficient strategy in clinical cancer theranostic field.

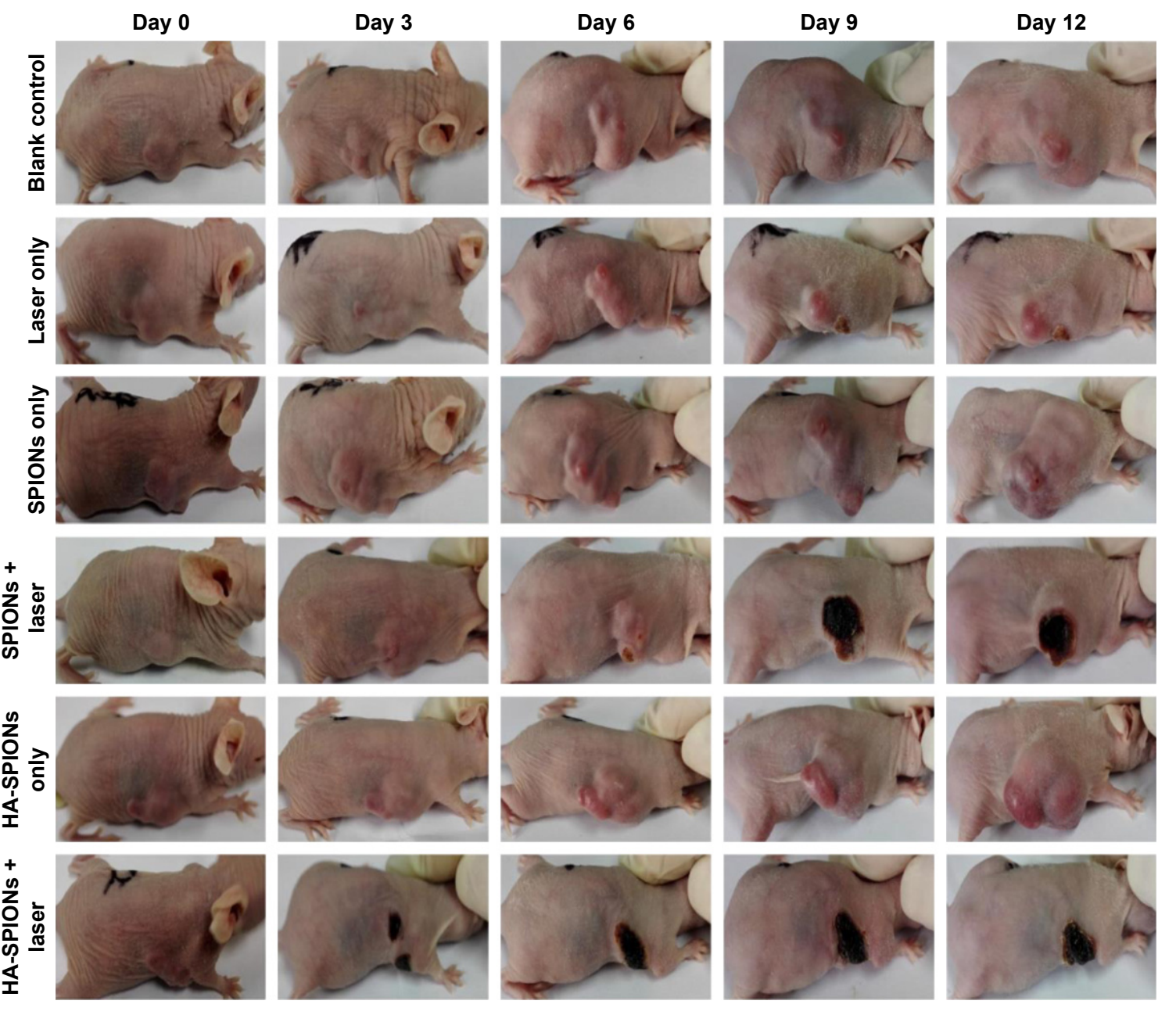

Figure 9 Representative photographs of tumors with and without laser irradiation.

Abbreviation: HA-SPION, hyaluronan-modified superparamagnetic iron oxide nanoparticle. 

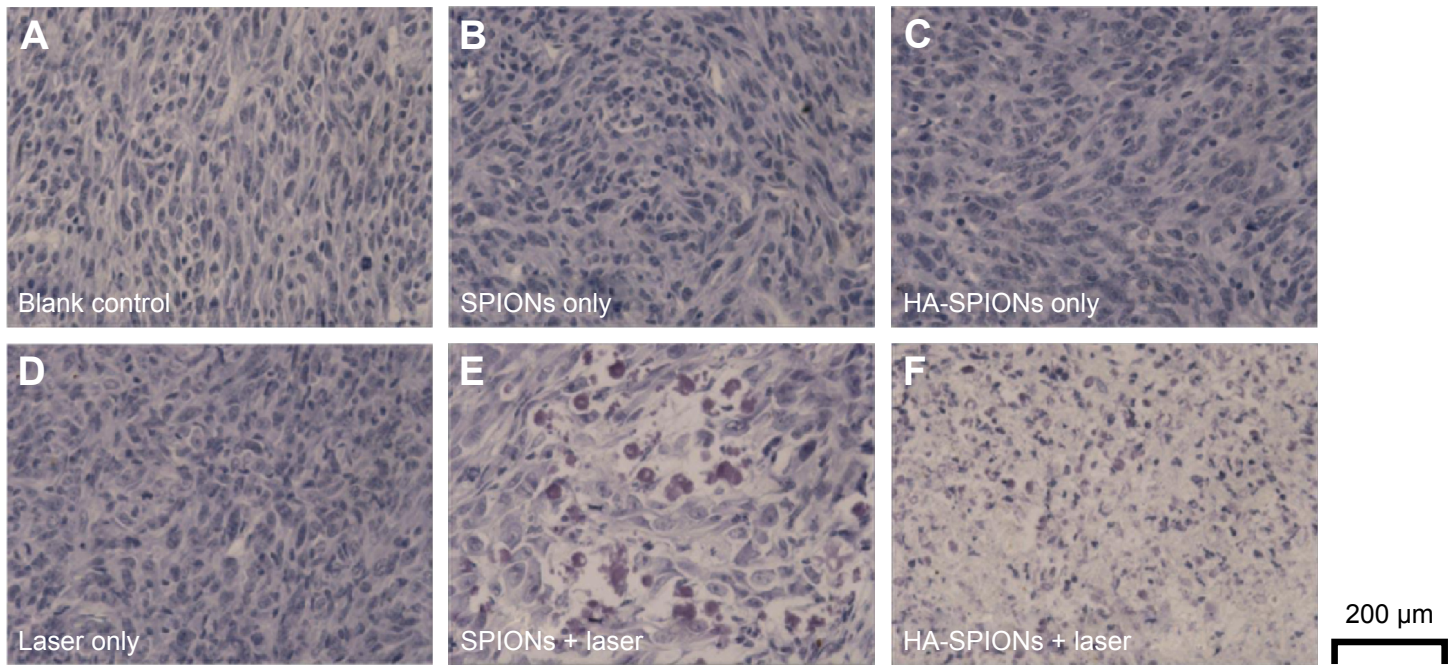

Figure 10 TUNEL-stained images of tumor tissues upon different experimental conditions: (A) control; (B) SPIONs only; (C) HA-SPIONs only; (D) laser only; (E) SPIONs combined with NIR laser treatments; and (F) HA-SPIONs combined with NIR laser treatments.

Abbreviations: HA-SPION, hyaluronan-modified superparamagnetic iron oxide nanoparticle; NIR, near-infrared; TUNEL, terminal deoxynucleotidyl transferase biotindUTP nick-end labeling.

\section{Acknowledgments}

This work is supported by the National Natural Science Foundation of China (81201087, 81571665, 21074152, 51273216, J1103305), 973 Program of China(2015CB755500), Guangzhou Key Technology R\&D Program (1563000477, 201607010038), and the Natural Science Foundation of Guangdong Province in China (10151006001000010, 2014A030313647). The authors thank Dr Hui-Kang Yang for his assistance in data processing.

\section{Disclosure}

The authors report no conflicts of interest in this work.

\section{References}

1. El-Sayed IH, Huang XH, El-Sayed MA. Selective laser photo-thermal therapy of epithelial carcinoma using anti-EGFR antibody conjugated gold nanoparticles. Cancer Lett. 2006;239(1):129-135.

2. Khlebtsov B, Zharov V, Melnikov A, Tuchin V, Khlebtsov N. Optical amplification of photothermal therapy with gold nanoparticles and nanoclusters. Nanotechnology. 2006;17(20):5167-5179.

3. Huff TB, Tong L, Zhao Y, Hansen MN, Cheng JX, Wei A. Hyperthermic effects of gold nanorods on tumor cells. Nanomedicine. 2007;2(1): $125-132$.

4. Takahashi H, Niidome T, Nariai A, Niidome Y, Yamada S. Photothermal reshaping of gold nanorods prevents further cell death. Nanotechnology. 2006;17(17):4431-4435.

5. Loo C, Lowery A, Halas N, West J, Drezek R. Immunotargeted nanoshells for integrated cancer imaging and therapy. Nano Lett. 2005;5(4): 709-711.

6. Hu M, Petrova H, Chen J, et al. Ultrafast laser studies of the photothermal properties of gold nanocages. J Phys Chem B. 2006;110(4):1520-1524.

7. Zhou M, Zhang R, Huang M, et al. A chelator-free multifunctional $[64 \mathrm{Cu}] \mathrm{CuS}$ nanoparticle platform for simultaneous micro-PET/CT imaging and photothermal ablation therapy. J Am Chem Soc. 2010;132(43): 15351-15358.

8. Kam NWS, O'Connell M, Wisdom JA, Dai HJ. Carbon nanotubes as multifunctional biological transporters and near-infrared agents for selective cancer cell destruction. Proc Natl Acad Sci U S A. 2005;102(33):11600-11605.
9. Chu MQ, Shao YX, Peng JL, et al. Near-infrared laser light mediated cancer therapy by photothermal effect of $\mathrm{Fe} 3 \mathrm{O} 4$ magnetic nanoparticles. Biomaterials. 2013;34(16):4078-4088.

10. Ahmed M, Douek M. The role of magnetic nanoparticles in the localization and treatment of breast cancer. Biomed Res Int. 2013;2013: 281230.

11. Unterweger H, Tietze R, Janko C, et al. Development and characterization of magnetic iron oxide nanoparticles with a cisplatin-bearing polymer coating for targeted drug delivery. Int J Nanomedicine. 2014; 9:3659-3676.

12. Dunn AW, Ehsan SM, Mast D, et al. Photothermal effects and toxicity of $\mathrm{Fe} 3 \mathrm{O} 4$ nanoparticles via near infrared laser irradiation for cancer therapy. Mater Sci Eng C Mater Biol Appl. 2015;46:97-102.

13. Yuan G, Yuan YJ, Xu K, Luo Q. Biocompatible PEGylated Fe3O4 nanoparticles as photothermal agents for near-infrared light modulated cancer therapy. International J Mol Sci. 2014;15(10):18776-18788.

14. Chen HW, Burnett J, Zhang FX, Zhang JM, Paholak H, Sun DX. Highly crystallized iron oxide nanoparticles as effective and biodegradable mediators for photothermal cancer therapy. J Mater Chem B. 2014;2(7):757-765.

15. Shen S, Wang S, Zheng R, et al. Magnetic nanoparticle clusters for photothermal therapy with near-infrared irradiation. Biomaterials. 2015;39:67-74.

16. Muthu MS, Leong DT, Mei L, Feng SS. Nanotheranostics - application and further development of nanomedicine strategies for advanced theranostics. Theranostics. 2014;4(6):660-677.

17. Bai LY, Yang XQ, An J, et al. Multifunctional magnetic-hollow gold nanospheres for bimodal cancer cell imaging and photothermal therapy. Nanotechnology. 2015;26(31):315701.

18. Xie J, Lee S, Chen XY. Nanoparticle-based theranostic agents. Adv Drug Deliv Rev. 2010;62(11):1064-1079.

19. Yang RM, Fu CP, Li NN, et al. Glycosaminoglycan-targeted iron oxide nanoparticles for magnetic resonance imaging of liver carcinoma. Mater Sci Eng C Mater Biol Appl. 2014;45:556-563.

20. Walia S, Sharma S, Markand Kulurkar P, Patial V, Acharya A. A bimodal molecular imaging probe based on chitosan encapsulated magneto-fluorescent nanocomposite offers biocompatibility, visualization of specific cancer cells in vitro and lung tissues in vivo. Int J Pharm. 2015;498(1-2):110-118. 


\section{Publish your work in this journal}

The International Journal of Nanomedicine is an international, peerreviewed journal focusing on the application of nanotechnology in diagnostics, therapeutics, and drug delivery systems throughout the biomedical field. This journal is indexed on PubMed Central, MedLine, CAS, SciSearch ${ }^{\circledR}$, Current Contents ${ }^{\circledR} /$ Clinical Medicine,

Journal Citation Reports/Science Edition, EMBase, Scopus and the Elsevier Bibliographic databases. The manuscript management system is completely online and includes a very quick and fair peer-review system, which is all easy to use. Visit http://www.dovepress.com/ testimonials.php to read real quotes from published authors.

Submit your manuscript here: http://www.dovepress.com/international-journal-of-nanomedicine-journal 